\title{
One-way-ness in the input-saving (Turing) machine
}

\author{
Alexandre de Castro* \\ Laboratório de Matemática Computacional, Centro Nacional de Pesquisa Tecnológica em Informática para a Agricultura (Embrapa \\ Informática Agropecuária), 13083-886, Brazil
}

\section{H I G H L I G H T S}

- We use an information heat engine to physically create a permutation operation.

- The mechanism relies on Bennett's algorithm of the reversible Turing machine.

- Reversion of the algorithm provoked erasure of information with gain of entropy.

- Erasure with gain of entropy causes a one-way (permutation) quantum gate.

- We showed that a cascade of two C-NOT gates in an adiabatic constraint is one-way.

\section{A R T I C L E I N F O}

\section{Article history:}

Received 24 April 2014

Available online 22 August 2014

\section{Keywords:}

One-way permutation

Computational complexity

Landauer's principle

$\mathrm{P}$ versus NP

Turing machine

\begin{abstract}
A B S T R A C T
Currently, a complexity-class problem is proving the existence of one-way permutations: one-to-one and onto maps that are computationally 'easy', while their inverses are computationally 'hard'. In what follows, we make use of Bennett's algorithm of the reversible Turing machine (quantum information heat engine) to perform a cascade of two controlledNOT gates to physically create a permutation operation. We show that by running this input-saving (Turing) machine backwards the critical inequality of Landauer's thermodynamic limit is reversed, which provokes the symmetry-breaking of the quantum circuit based on two successive controlled-NOT quantum gates. This finding reveals that a permutation of controlled-NOT gates becomes one-way, provided that adiabatically immersed in a heat bath, which determines the condition of existence of a thermodynamically noninvertible bijection in polynomial-time, that would otherwise be mathematically invertible. This one-way bijection can also be particularly important because it shows nonlinearities in quantum mechanics, which are detectable by watching that the mathematical reversibility of controlled-NOT gates does not work physically.
\end{abstract}

(C) 2014 Elsevier B.V. All rights reserved.

\section{Introduction}

Inverse problems are frequently encountered in a wide range of fields, and particularly, hard-to-invert ones are commonly exploited in cryptology-related realms. Currently, data-security theory has been heavily based on an unproven one-way-ness proposition of computational paths, which holds that there must be a one-to-one correspondence for which the forward calculation is feasible, whereas reconstructing the input state from the output state is computationally impracticable.

\footnotetext{
* Tel.: +55 1932115859 .

E-mail address: alexandre.castro@embrapa.br.
} 

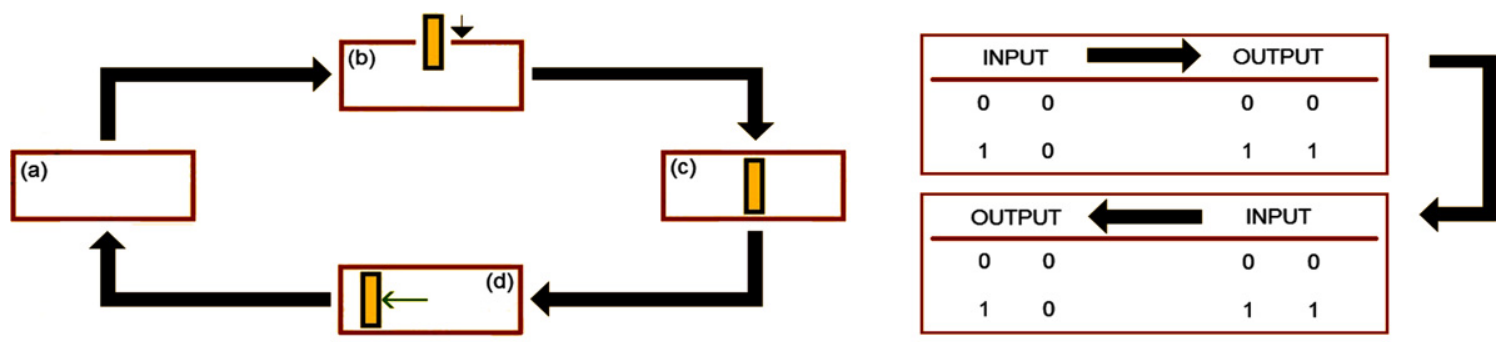

Fig. 1. Calculating forward in two non-commutative stages of Bennett's algorithm of Szilárd's one-molecule engine, in Ref. [15], Bennett models an electronic analog of this device in which the position of a diamagnetic molecule in the measured system is detected when it flips the state of a bistable ferromagnet (measuring system). This reversible Turing machine saves its input, thereby ensuring a global 1:1 relation between the initial and the final states, even when the function being computed is many-to-one. All of the thermodynamics of the forward calculation can then be summarized in a noncommutative transition from non-random data to random data and from random data to non-random data again. In this state transition, the correlation process is represented by the controlled-NOT quantum gate, as shown in the right side. In the first stage, the insertion of the partition, shown in (a $\rightarrow \mathrm{b}$ ), corresponds to the randomization of the measuring system without an associated thermodynamic cost. In the next stage, an "erasure" of the measuring system occurs to restore the standard state. The decrease in the entropy in the measuring system provoked by the data erasure generates a minimum increase in the entropy, equal to $k_{B} \log (2)$ per bit-flipping, in the measured system. This causes an isothermal expansion in the measured system, as shown in $(c \rightarrow d \rightarrow a)$.

In the last few years, the idea has been widely accepted that the existence of a one-way bijection [1-3] proves that the complexity-classes P (deterministic polynomial-time) and NP (nondeterministic polynomial-time) are not equal $[4,5]$. Such a one-way-ness conjecture arises automatically from the self-referential nature of $\mathrm{P}=$ ?NP problem [6], because in the self-reference paradoxes (see Cantor's theorem and Russell's paradox), a one-to-one correspondence does not behave as a bijection $[7,8]$.

However, while all mathematical efforts to prove the existence of one-way bijections have been doomed to failure, the assumption has gained particular importance that the complexity-class problems can be linked to physical constraints rather than purely mathematical limitations [9-11].

Based on this perspective, we present here a new approach that connects one-way permutations to the thermodynamic bounds of computation. In what follows, it is shown that, although it is well known in cryptosystem theory that one-to-one correspondences can be mathematically invertible in polynomial-time with a zero-failure probability, a quantum circuit of logical gates that map its target bit onto itself [12] encounters an entropy constraint to be physically inverted if immersed in a heat bath (adiabatic).

This paper is organized as follows. First, an overview of the well-known Bennett's algorithm of the reversible Turing machine is presented, which is an input-saving machine the transition function of which maps its logic-input state to the output unchanged $[13,14]$. Second, I will explore the entropy bounds of a full computational cycle by running (in reverse) Bennett's algorithm to prove that the computational path to thermodynamically undo the cycle cannot be performed in polynomial-time.

\section{Theoretical background}

Let us distinguish a couple of logical structures in Bennett's Turing machine, namely, a binary memory and a measured system, the logical states of which are ruled by a controlled-NOT operator (C-NOT quantum gate), wherein the memory is the target bit. Such a controlled-NOT operation is conceived to be mathematically embodied in an information heat engine, the feedback controller of which manipulates the measured system (an adiabatic box) based on its thermal fluctuations into its logical memory (a heat reservoir) to restore its standard state [13-23].

\subsection{One-to-two relation}

To measure and restore the standard state of the memory, Bennett's algorithm, fundamentally, utilizes two noncommutative stages. Initially, a removable partition is inserted (with no thermodynamic work) into the middle of the adiabatic box, resulting in the splitting of the memory into a twofold state. Before the insertion, as shown in Fig. 1(a) and (b), the C-NOT quantum gate operator stores the memory state in the target bit " 0 " with a probability equal to unity, whereas the control bit is in a " 0 " or "1". After the insertion of the partition, as shown in Fig. 1(c), the logical state of the measuring system becomes perfectly correlated with the logical state of the measured system. This operation does not result in any heat exchange with the measured system and corresponds to the target bit being flipped whether the control bit is " 1 " in the C-NOT quantum gate. In other words, the measurement (acquisition of information from Bennett's conception) induces a randomization of the memory state with a bit equally likely to be " 0 " or "1". 


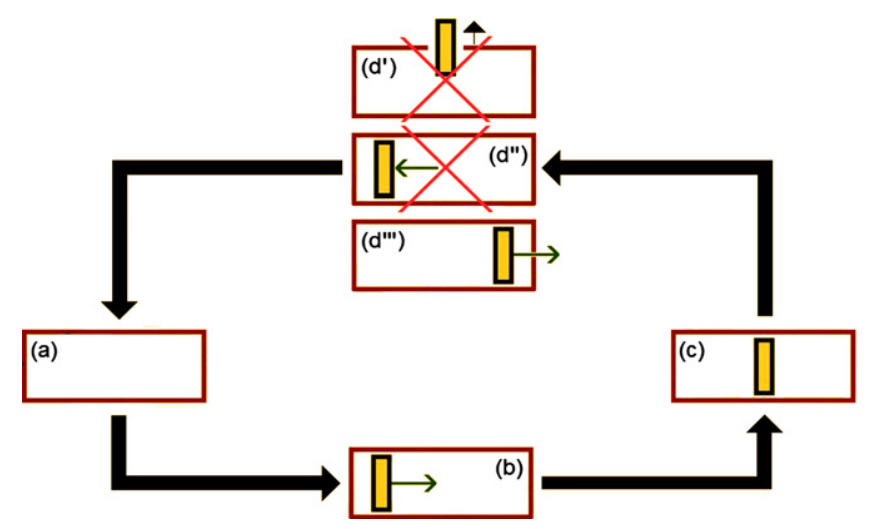

\begin{tabular}{|c|c|c|c|}
\hline \multicolumn{2}{|c|}{ INPUT } & \multicolumn{2}{|c|}{ OUTPUT } \\
\hline 0 & 0 & 0 & 0 \\
\hline 1 & 0 & 1 & 1 \\
\hline \multicolumn{2}{|c|}{ OUTPUT } & \multicolumn{2}{|c|}{ INPUT } \\
\hline 0 & 0 & 0 & 0 \\
\hline 1 & 0 & 1 & 1 \\
\hline
\end{tabular}

Fig. 2. Calculating backward the global 1:1 relation in two non-commutative stages. Whereas in (a-b-c) the net change in entropy over the course of the operation must be zero, the backward cycle is only completed if the entropy decreases in the measured system. Running forward, Maxwell's demon trivially returns the input state with $\Delta S>0$, as shown in Fig. 1. However, running the backward calculation cannot thermodynamically recover the input insofar as the C-NOT operation in the last branch of the cycle induces a $\Delta S<0$ in the measured system. Because Landauer's principle prevents a data erasure occurring at zero thermodynamic cost, as shown in $\left(\mathrm{d}^{\prime}\right)$, and because moving the partition toward the far left site corresponds to the forward calculation, as shown in $\left(\mathrm{d}^{\prime \prime}\right)$, algorithmically, there remains only to move the partition toward the far right site, as shown in $\left(\mathrm{d}^{\prime \prime \prime}\right)$. However, such a $\left(\mathrm{c} \rightarrow \mathrm{d}^{\prime \prime \prime} \rightarrow \mathrm{a}\right)$ procedure provokes an entropy decrease in the adiabatic box, which implies a time reversal for running the backward cycle. Consequently, a computational path that undoes the cycle cannot be performed in polynomial-time.

\subsection{Two-to-one relation}

In the next stage, a logical merging of two states into one should occur while performing a loop closing. If we merge data from a symmetric double-well memory state, then there must be a change in some other macroscopic variable of the ensemble. Liouville's theorem requires that this change be a volume-preserving operation, for which the state space should remain invariant under the transformation, provided that the region available to the logical degrees of freedom is reduced by a factor of two and that the region available to the non-information bearing degrees of freedom is doubled.

The thermodynamics of this logical operation corresponds to an isothermal expansion in the adiabatic box, similar to compression of a one-molecule gas by a piston in the symmetric Szilárd's engine, in which the frictionless partition moves quasi-statically toward the far left side of the box in such a way that the measured system becomes detached from the measuring system whenever the partition reaches the end of the pathway, as shown in Fig. 1(d). At the end of the isothermal expansion, the computation path is complete, and the memory is restored to its standard state " 0 " with a probability equal to unity.

This restore-to-zero operation detaches the symmetric binary memory from the measured system, working in the same manner as an information erasure. By Landauer's principle, this erasure is accompanied by heat generation into the adiabatic box, so that at the end of the full computational path, the net balance of entropy results in a minimal increase of entropy, $\Delta S \geq k_{B} \log (2)$, in the measured system, where $k_{B}$ is the Boltzmann constant [12-23]. As a consequence, the entropy increase of the measured system is compensated by an entropy decrease of the random data, rendering the operation as a whole thermodynamically reversible.

\subsection{Origin of the inverse problem}

As the erasure is thermodynamically reversible, one can run it in reverse and rebuild the memory to the two equally probable states in a one-to-two relation again (as Bennett originally performed in Ref. [13]). The thermodynamics of this reversal operation corresponds to the isothermal compression in the measured system shown in Fig. $2(\mathrm{a} \rightarrow \mathrm{b} \rightarrow \mathrm{c})$, in which the partition slowly slides from the far left side $(V)$ toward the middle of the box $\left(\frac{V}{2}\right)$, rebuilding the target state to either " 0 " or " 1 ". As a result, the previous amount of heat generated in the measured system returns to the memory, and the heat extracted in the pathway is once again converted into thermodynamic work $[20,23]$.

In this way, the combination of conducting an erasure followed by its reversal results in no net entropy change, as shown in Eq. (1). Thereby, the procedure shown in Fig. 1(c $\rightarrow d \rightarrow$ a), combined with the procedure shown in Fig. 2(a $\rightarrow$ b $\rightarrow c)$, is thermodynamically equivalent to the correlation operation shown in Fig. $1(\mathrm{a} \rightarrow \mathrm{b} \rightarrow \mathrm{c})$.

$$
\int_{\frac{V}{2} \rightarrow V} K_{B} \frac{\mathrm{d} V}{V}+\int_{\frac{V}{2} \leftarrow V} K_{B} \frac{\mathrm{d} V}{V}=0 .
$$


The trouble is that an erasure followed by its reversal does not constitute a complete cycle because the memory state acquires another random bit $[13,23]$. Once the forward computation of Bennett's algorithm restores the target bit "0", then a closing of the backward cycle (global 1:1 relation in reverse) is still required to undo the correlation shown in Fig. 2(c).

\section{Entropy constraint}

Let us show that there exist three alternatives to close the backward cycle and attaining the target bit " 0 ". First, one may dissipationlessly remove the partition, as Fig. $2\left(\mathrm{~d}^{\prime}\right)$ displays. However, this would be like data being erased in the memory at zero thermodynamic cost, which Landauer's principle prevents [20-24]. Second, one may quasi-statically move the partition toward the far left side of the box, as Fig. $2\left(\mathrm{~d}^{\prime \prime}\right)$ displays. However, this would increase the entropy in the measured system, which would result in further forward computations, such as the procedure accomplished in the logical merging shown in Fig. 1(d), and not backward as we are pursuing. Then, the only remaining option is to quasi-statically move the partition toward the far right side of the box, as Fig. $2\left(\mathrm{~d}^{\prime \prime \prime}\right)$ displays.

By moving the partition toward the far right side of the box, the measured system is detached from the measuring system, and the memory returns to the standard state again. Algorithmically, this isothermal compression of the measured system is the unique admissible subroutine to attain (in reverse) the target bit " 0 ". However, such a subroutine leads to a thermodynamic constraint (as described in the Methods section) because it causes an entropy decrease in the adiabatic box $(\Delta S<0)$. As a result, the cycle in reverse cannot be completed in polynomial-time, once entropy is the only physical quantity that requires an arrow of time.

\subsection{Methods}

Let us again consider the feedback-control engine shown in Fig. 1. Before the measurement, the memory is in the standard state " 0 ". The measured system then establishes a perfect correlation with the measuring system, and the target bit is flipped in the C-NOT quantum gate, so that the change in the memory state, $M$, can be represented by:

$$
\frac{\mathrm{d}}{\mathrm{d} t} M=\delta\left\langle I_{t}, M\right\rangle,
$$

where $I_{t}$ is the measurement outcome, or equivalently, the information per unit time obtained by measurement, and $\delta$ is a normalized matching metric (this metric is the same as a key-lock model that we have used in previous works, see Refs. [25$27])$. Note that the feedback control in Bennett's Turing machine establishes a perfect correlation $(\delta=1)$, but in a not perfect correlation between the measuring system and the measured system, $0 \leq \delta \leq 1$.

Now, note that the restore-to-zero (forward) operation, depicted in Fig. 1(c $\rightarrow d \rightarrow$ a), is caused by a negative feedback between the measuring system and the measured system because the region available to the logical degrees of freedom is reduced by a factor of two, whereas the region available to the non-information bearing degrees of freedom is doubled in an isothermal expansion of the measured system. In the restore-to-zero (backward) operation, depicted in Fig. 2( $c \rightarrow d^{\prime \prime \prime} \rightarrow$ a), however, a positive feedback between the measuring system and the measured system occurs because the compression of the two states into one in memory occurs at the expense of an isothermal compression of the measured system.

As the measured system must restore the standard state " 0 " when one runs the forward cycle as well as the backward, the dynamics of the control mechanism can then be written according to the negative and positive correlations between $I_{t}$ and $M$, as follows:

$$
\left[\frac{\mathrm{d}}{\mathrm{d} t} M\right]_{\text {restore-to-zero }}= \begin{cases}+I_{t} M ; & \text { for negative feedback } \\ -I_{t} M ; & \text { for positive feedback. }\end{cases}
$$

Considering that, in the detachment operation shown in Fig. 2(c $\rightarrow \mathrm{d}^{\prime \prime \prime} \rightarrow$ a), the C-NOT quantum gate flips the target bit once only, the erasure in reverse is then defined from Eq. (3) as follows:

$$
-\left[I_{t} \Delta t\right]_{\text {positive feedback }}=\int_{M_{i}}^{M_{f}} \frac{\mathrm{d} M}{M}=\log \left(1+\frac{\Delta M}{M_{i}}\right),
$$

where $\Delta t$ is the time elapsed in the erasure, $M_{f}$ and $M_{i}$ are final and initial memory states, respectively, and $\frac{\Delta M}{M_{i}}$ is the relative change in the memory register when the information is discarded (Weber-like ratio).

Still, if the input $I_{t}$ corresponds to a one bitwise operation per unit time, one can then define $I_{t} \Delta t \equiv I$. Furthermore, once the restore-to-zero operation provokes a loss of randomness by a merge of two logical states into one in memory (relative change equal to $-50 \%)$, the $\frac{\Delta M}{M_{i}}$ term in Eq. (4) assumes a value equal to $-\frac{1}{2}$. Thereby, the information discarded during the erasure in reverse depicted in Fig. $2\left(\mathrm{c} \rightarrow \mathrm{d}^{\prime \prime \prime} \rightarrow\right.$ a) is equal to $I=\log (2)$, in natural unit. Otherwise, the information discarded during the forward erasure depicted in Fig. $1(\mathrm{c} \rightarrow \mathrm{d} \rightarrow \mathrm{a})$ is equal to $I=-\log (2)$. 
Let us also remember that the information engine of Bennett's algorithm is immersed in an isothermal heat bath. Thus, the information erased can be subjected to a thermal treatment by a scaling factor of $K_{B} T$, so that the control mechanism reaches the maximum efficiency.

In this way, when the cycle runs in reverse, $I$ is converted into free energy, which provokes an entropy increase in memory equal to $\Delta S=K_{B} \log (2)$ per information erased. Note that for a not perfect correlation $(0 \leq \delta \leq 1)$, the inequality $\Delta S \leq K_{B} \log (2)$ appears. This is in contrast to the forward calculation, in which the erasure of one bit of information provokes an entropy decrease in the memory equal to $\Delta S \geq-K_{B} \log (2)$.

As Bennett's Turing machine operates adiabatically, such an erasure in reverse performed with an entropy increase in memory must be compensated by an entropy decrease of the measured system (see more detail in the Appendix). This means that Landauer's principle in reverse about information erasure violates the second law of thermodynamics and provokes time reversal in the measured system because entropy is the only physical quantity that requires an arrow of time [21,28-33]. Thus, an input-saving machine cannot complete its backward cycle in polynomial-time, which implies that its controlledNOT-based quantum circuit is one-way inside adiabatic thresholds. This result shows that there is a physically-motivated symmetry group, namely, a symmetry-protected automorphism in the input-saving machine.

Thereby, an important point addressed in this paper is that an entropic one-way-ness implies existence of one-way permutation. To show this, we used the controlled-NOT quantum gate that is a one-to-one and onto map because its action is undone if a second controlled-NOT gate is applied. This permutation is straightforward to verify by computing the square of the matrix representation of the C-NOT gate.

In this work, we showed that a sequence of two successive controlled-NOT-gates becomes a hard-to-invert bijection, provided that confined in adiabatic thresholds. In other words, we showed that if two successive controlled-NOT gates are performed in entropic boundary conditions, we get a one-way permutation. Thereby, a quantum circuit based on two successive controlled NOT-gates is invertible if computed without an entropy constraint, but non-invertible if computed with an entropy constraint (one-way boundary condition). Consequently, the T-asymmetry of the entropy (one-way function) implies directly existence of a one-way permutation, which shows that the complexity-classes $\mathrm{P}$ and NP are not equal for the involutory function $\mathrm{NP}(\mathrm{NP}(\mathrm{P}))=\mathrm{P}$.

\section{Acknowledgment}

The author would especially like to thank Edgard H. dos Santos for the fruitful discussions and numerous valuable comments without which this paper would not be written.

\section{Appendix}

Here, we detail key steps in the derivation of the result presented in the Methods section.

Balance of entropy. To concretely know the "amount of time" that takes for computing a logic operation, one must dip the logic gate in a heat bath and, then, computing the balance of entropy involved in the complete operation, once entropy is the only quantity that requires a particular direction for time.

Considering that the feedback control mechanism (controlled NOT quantum gate), shown in Figs. 1 and 2, is embedded in an adiabatic heat bath, then, any entropy increase in the measuring system to perform the quantum circuit must be compensated by an entropy decrease of the measured system, and vice versa.

Thus, the balance of entropy in the complete back-and-forth calculation is as follows:

\begin{tabular}{|c|c|}
\hline \multicolumn{2}{|l|}{ Forward cycle } \\
\hline Correlation $^{\mathrm{a}}$ (one-to-two relation) $\left(\begin{array}{ll}0 & 0 \\
1 & 0\end{array}\right) \rightarrow\left(\begin{array}{ll}0 & 0 \\
1 & 1\end{array}\right)$ & $\begin{array}{l}\Delta S_{\text {measuring system }}=0 \\
\Delta S_{\text {measured system }}=0\end{array}$ \\
\hline $\begin{array}{l}\text { Erasure } \mathrm{b}^{\mathrm{b}} \text { (two-to-one relation) Feedback negative } \\
\left(\begin{array}{ll}0 & 0 \\
1 & 1\end{array}\right) \rightarrow\left(\begin{array}{ll}0 & 0 \\
1 & 0\end{array}\right)\end{array}$ & $\begin{array}{l}\Delta S_{\text {measuring system }}=\frac{1}{\delta} \int_{M_{i}}^{M_{f}} K_{B} \frac{\mathrm{d} M}{M} \Rightarrow \\
\Delta S_{\text {measuring system }} \geq K_{B} \log \left[1+\left(\frac{\Delta M}{M_{i}}=-\frac{1}{2}\right)\right] \\
\Delta S_{\text {measuring system }} \geq-K_{B} \log (2) \\
\Delta S_{\text {measured system }} \geq \int_{V}^{V} K_{B} \frac{\mathrm{d} V}{V} \geq+K_{B} \log (2)\end{array}$ \\
\hline $\begin{array}{l}\text { a The balance of entropy of the (forward) correlation bet } \\
\text { corresponds to the operation of insertion of the partition } \\
\text { not result in any heat exchange with the measured syste } \\
\text { b This erasure operation, as shown in Fig. } 1(\mathrm{c} \rightarrow \mathrm{d} \rightarrow \mathrm{a} \text { ), } \\
\text { system, and the entropy decrease in memory generates a } \\
\text { the end of the full (forward) computational path, the net } \\
\text { the measured system equal to } \Delta S \geq k_{B} \log (2) \text {. This oper } \\
\text { and outputs the state " } 0 \text { ". }\end{array}$ & $\begin{array}{l}\text { heasuring system and measured system } \\
\text { in Fig. } 1(\mathrm{a} \rightarrow \mathrm{b} \rightarrow \mathrm{c}) \text {. This operation does } \\
\text { ine measuring system from the measured } \\
\text { entropy into the adiabatic box, so that at } \\
\text { scard of information takes two input states, }\end{array}$ \\
\hline
\end{tabular}




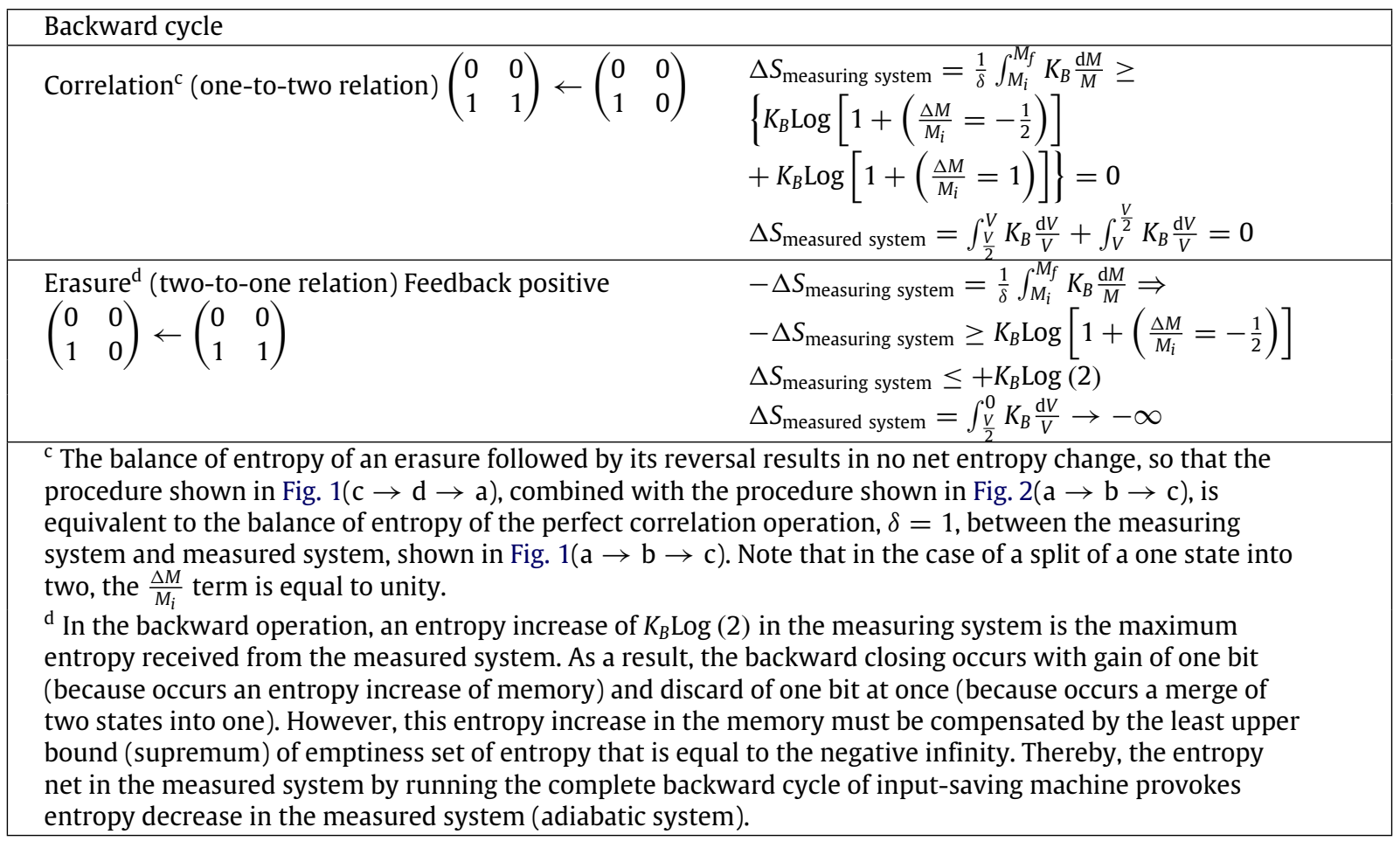

\section{References}

[1] A.A. Razborov, S. Rudich, Natural proofs, J. Comput. System Sci. 55 (1997) 24.

[2] L.A. Levin, The tale of one-way functions, Probl. Inf. Transm. 39 (1) (2003) 92.

[3] O. Goldreich, The Foundations of Cryptography. Basic Tools, Vol. 1, Cambridge University Press, 2006.

[4] S. Cook, The complexity of theorem proving procedures, in: Proceedings of the Third Annual ACM Symposium on Theory of Computing, 1971, pp. 151-158.

[5] R.J. Lipton, K.W. Regan, People, Problems, and Proofs: Essays from Gödel's Lost Letter: 2010, Springer, New York, 2013.

[6] S. Aaronson, Is P versus NP formally independent? Bull. Eur. Assoc. Theor. Comput. Sci. EATCS 81 (2003) 109-136.

[7] G. Priest, Beyond the Limits of Thought, Clarendon Press, Oxford, 2003.

[8] H. Gaifman, Naming and diagonalization, from Cantor to Gödel to Kleene, Logic J. IGPL 14 (2006) 709.

[9] W.H. Zurek, Thermodynamic cost of computation, algorithmic complexity and the information metric, Nature 341 (1989) 119.

[10] S. Aaronson, NP-complete problems and physical reality, SIGACT News 36 (1) (2005).

[11] S. Aaronson, Why philosophers should care about computational complexity, in: B.J. Copeland, C. Posy, O. Shagrir (Eds.), Computability: Gödel, Turing, Church, and Beyond, MIT Press, Cambridge, MA, 2012.

[12] R. Raussendorf, H.J. Briegel, A one-way quantum computer, Phys. Rev. Lett. 86 (22) (2001) 5188.

[13] C.H. Bennett, R. Landauer, The fundamental physics limits of computation, Sci. Am. 253 (1985) 48.

[14] C.H. Bennett, Time/space trade-off for reversible computation, SIAM J. Comput. 20 (4) (1989) 78.

[15] C.H. Bennett, The thermodynamics of computation: a review, Internat. J. Theoret. Phys. 21 (1982) 905.

[16] T. Sagawa, Thermodynamics of information processing in small systems, Progr. Theoret. Phys. 127 (2012) 1.

[17] C.H. Bennett, Demons, engines and the second law, Sci. Am. 257 (1987) 108.

[18] O.J.E. Maroney, Information processing and thermodynamic entropy, in: E.N. Zalta (Ed.), The Stanford Encyclopedia of Philosophy (Fall 2009 Edition), http://plato.stanford.edu/archives/fall2009/entries/information-entropy/.

[19] M.B. Plenio, V. Vitelli, The physics of forgetting: Landauer's erasure principle and information theory, Contemp. Phys. 42 (2001) 25.

[20] O.J.E. Maroney, Generalizing Landauer's principle, Phys. Rev. E 79 (2009) 031105.

[21] O.J.E. Maroney, Does a computer have an arrow of time? Found. Phys. 40 (2010) 205.

[22] C.H. Bennett, Notes on the history of reversible computation, IBM J. Res. Dev. 32 (1988) 16.

[23] C.H. Bennett, Notes on Landauer's principle, reversible computation, and Maxwell's demon, Stud. Hist. Philos. Modern Phys. 34 (2003) 501.

[24] R. Landauer, Irreversibility and heat generation in the computing process, IBM J. Res. Dev. 5 (1961) 183.

[25] A. Castro, A network model for clonal differentiation and immune memory, Physica A (2005) 408.

[26] A. Castro, The thermodynamic cost of fast thought, Minds Mach. 23 (2013) 473.

[27] A. Castro, A Shannon-like solution for the fundamental equation of information science, in: B.K. Tripathy, D.P. Acharjya (Eds.), Global Trends in Intelligent Computing Research and Development, IGI Global, 2014, pp. 516-524.

[28] R. Landauer, Statistical physics of machinery: forgotten middle-ground, Physica A 194 (1993) 551.

[29] R. Landauer, Information is a physical entity, Physica A 263 (1999) 63.

[30] L. del Rio, J. Åberg, R. Renner, O. Dahlsten, V. Vedral, The thermodynamic meaning of negative entropy, Nature 474 (2011) 61.

[31] J. Hartle, T. Hertog, Arrows of time in the bouncing universes of the no-boundary quantum state, Phys. Rev. D 85 (2012) 103524.

[32] S. Hawking, A Briefer History of Time (with Leonard Mlodinow), Bantam Dell, 2008.

[33] S. Toyabe, T. Sagawa, M. Ueda, E. Muneyuki, M. Sano, Experimental demonstration of information-to-energy conversion and validation of the generalized Jarzynski equality, Nat. Phys. 6 (2010) 988. 